ThE Astrophysical Journal, 337:720-729, 1989 February 15

(C) 1989. The American Astronomical Society. All rights reserved. Printed in U.S.A

\title{
SLOW EXPANSION OF THE SHELL OF THE RECURRENT NOVA T PYXIDIS AND DETECTION OF A FAINT EXTENDED ENVELOPE
}

\author{
Michael M. Shara ${ }^{1,2,3}$ \\ Space Telescope Science Institute \\ ANTHONY F. J. MOFFAT ${ }^{1,3}$ \\ Université de Montreal \\ ROBERT E. WILLIAMS \\ Cerro Tololo Inter-American Observatory and National Optical Astronomy Observatories \\ AND \\ JUDITH G. COHEN \\ Palomar Observatory, California Institute of Technology \\ Received 1987 March 19; accepted 1988 August 2
}

\begin{abstract}
Deep CCD images of the recurrent nova T Pyx have revealed a faint, extended $\mathrm{H} \alpha+[\mathrm{N}$ II $]$ halo twice as large as the previously detected shell. An [O III] image of T Pyx shows a smooth, small shell.

Comparison of 1980 and 1985 images of the $\mathrm{H} \alpha+[\mathrm{N}$ II $]$ shell show an expansion of less than $10 \%$. If the bright, inner shell is due to the 1966 eruption, it should have expanded $\sim 36 \%$ from 1980 to 1985 (assuming uniform shell expansion). We rule out the possibility of the $\mathrm{T}$ Pyx shell being associated with a planetary nebula-type ejection for two reasons: the shell mass is less than $10^{-4} M_{\odot}$, and the shell expansion velocity is $\sim 350 \mathrm{~km} \mathrm{~s}^{-1}$.

This expansion velocity is much slower than the $850 \mathrm{~km} \mathrm{~s}^{-1}$ and $2000 \mathrm{~km} \mathrm{~s}^{-1}$ velocities reported by Catchpole (1969) during the 1966 outburst.

If the $10^{\prime \prime}$ diameter shell is from the 1966 outburst, then the ejecta have given up most of their bulk kinetic energy by interaction with circumstellar matter or significant amounts of (now visible) low-velocity material were ejected during the last outburst, or both. The lack of strong [O I] $\lambda 6300$ and [S II] $\lambda \lambda 6717,34$ emission lines argues against much shock interaction at the present era, and, indirectly, for the 1944 identification of the $10^{\prime \prime}$ shell, while thermonuclear runaway nova models support the multiple-velocity idea. A point-spread function subtracted from an $\mathrm{H} \alpha+[\mathrm{N}$ II $]$ image of $\mathrm{T}$ Pyx has revealed a $2^{\prime \prime}$ radius ring around the central star. This may be the ejecta from the 1966 eruption.

The photoionized shell gas implies that the central star should be UV-bright. High resolution Hubble Space Telescope imaging observations of the next T Pyx eruption might yield early detection of light echoes.
\end{abstract}

Subject headings: stars: binaries — stars: variables - stars: novae - nebulae: internal motions

\section{INTRODUCTION}

Recurrent novae (RNs) are the rarest class of cataclysmic variables, brightening dramatically for a few weeks every few decades. Although RN outbursts resemble those of classical novae, the latter are believed to have eruptions separated by thousands of years (Patterson 1984; Shara et al. 1986). Why the outbursts are similar and the recurrence times are so different is an important question any successful theory of cataclysmics must answer.

In a recent exhaustive review of the subject of recurrent novae, Webbink et al. (1987) concluded that there are only four certain members of the class: RS Oph, T Cor Bor, U Sco, and $T$ Pyx. Furthermore, only the U Sco and T Pyx outbursts appear to be thermonuclear runaways, and thus genuinely related to classical novae. T Pyx is considerably brighter than

${ }^{1}$ Guest Observer, Anglo-Australian Observatory.

${ }^{2}$ Guest Investigator, The Interactive Astronomical Data Analysis Facility, the Laboratory for Astronomy and Solar Physics, NASA/Goddard Space Flight Center.

${ }^{3}$ Visiting Astronomer, Cerro Tololo Inter-American Observatory of the National Optical Astronomy Observatories, operated by AURA, Inc. under contract with the National Science Foundation.
U Sco (when both are at minimum) and probably considerably closer (Webbink et al. 1987). A better understanding of T Pyx is therefore central to further insight into the nature of recurrent novae, and to what differentiates them from classical novae.

The recurrent nova T Pyxidis has five recorded outbursts to its credit (in 1890,1902,1920,1944, and 1966), all of which were very similar photometrically (Mayall 1967). The slow rise and decline of the optical light curves indicate that the luminosity of T Pyx at maximum cannot greatly exceed the Eddington limit (Webbink et al. 1987).

Catchpole's (1969) spectroscopic observations showed “principal ejecta" absorption features at $V \approx 850 \mathrm{~km} \mathrm{~s}^{-1}$, and other absorption indicative of velocities up to $\sim 2000 \mathrm{~km} \mathrm{~s}^{-1}$. While Catchpole (1969) found no spectroscopic evidence of circumstellar matter, narrow-band $\mathrm{H} \alpha$ imaging of $\mathrm{T}$ Pyx by Duerbeck and Seitter (1979) and Williams (1981, 1982) has revealed a shell with diameter $\approx 10^{\prime \prime}$ surrounding the star.

The shell expansion velocity, angular size, and the time since eruption should, in principle, be sufficient to determine a distance. Unfortunately, it is not obvious which outburst is responsible for the shell, nor which ejection velocities should be used in calculating the nova's distance. No other recurrent 
nova has a detected shell or a well determined distance. Thus, as emphasized above, any improvement in our knowledge of recurrent nova absolute magnitudes, accretion rates, shell masses (or any parameter which demands knowledge of the distance) is most likely to come from T Pyx.

In $\S$ II and III we report on new $\mathrm{H} \alpha$ narrow-band CCD imaging of T Pyx and the detection of a very faint, extended $\mathrm{H} \alpha$ halo surrounding the already known shell. We also display an [O III] image containing an emitting shell with a morphology different from that of the $\mathrm{H} \alpha$ shell and we report measurements of the $\mathrm{H} \alpha$ shell expansion which rule out the 1966 eruption date for the shell origin, assuming uniform expansion. We propose in $\S$ IV that the observed shell is slowly moving, solar abundance ejecta which is photoionized. The next eruption of T Pyx should be closely monitored by the Hubble Space Telescope to look for light echoes. In $\S \mathrm{V}$ we briefly summarize our results.

\section{OBSERVATIONS}

R. E. W. imaged T Pyx on 198014 March in excellent seeing at the prime focus of the CTIO $4 \mathrm{~m}$ telescope. A photographic reproduction of a 75 minute exposure on a baked 098-04 plate taken through an $\mathrm{H} \alpha+[\mathrm{N}$ II] interference filter has already been published (Williams 1982). The area on the original plate around T Pyx has now been digitized (50 $\mu \mathrm{m}$ aperture, $28 \mu \mathrm{m}$ steps) with the PDS microdensitometer of the Goddard Space Flight Center's Interactive Astronomical Data Analysis Facility.

On 1985 May 21 M. M. S. and A. F. J. M. imaged T Pyx with the prime focus RCA CCD camera of the AngloAustralian Observatory $3.9 \mathrm{~m}$ telescope. Images were taken with a $6563 \AA \mathrm{H} \alpha$ filter (50 $\AA$ FWHM); and a broad-band $\mathrm{R}$ filter. Exposure times were 30 minutes for the narrow-band exposure and $100 \mathrm{~s}$ for the broad-band exposure.

On 1986 January 19 M. M. S. and A. F. J. M. imaged T Pyx with the $0.9 \mathrm{~m}$ telescope + RCA CCD of the Cerro Tololo Interamerican Observatory. A $3600 \mathrm{~s}$ image taken through an [O III] $\lambda 5007$ filter $(20 \AA$ FWHM) and a 120 s B image were obtained. All the image data reductions and image processing reported in $\S$ III were performed at ST ScI.

J. G. C. obtained two 30 minute spectra of the T Pyx shell on the night of 1987 April 17, with the Hale $5 \mathrm{~m}$ telescope and double spectrograph. The $1^{\prime \prime}$ seeing on that night yielded a 2 pixel FWHM, corresponding to a velocity resolution of $75 \mathrm{~km}$ $\mathrm{s}^{-1}$ from the $0.811 \AA$ pixel $^{-1}$ dispersion. The spectra were flattened, and night sky lines subtracted out at Caltech.

\section{SHELL IMAGES AND SPECTRA}

\section{a) Images}

The digitized image of T Pyx (from the 1980 CTIO H $\alpha$ plate) is shown in Figure $1 a$ beside the 1985 AAT CCD image (Fig. $1 b$ ). The images are displayed at the same scale, and the CCD image has been assigned a lookup table to highlight the same features as the digitized plate. It is immediately and remarkably apparent how little the shell's appearance has changed in five years. We quantify this point below.

In Figure $2 a$ we again display the $\mathrm{H} \alpha \mathrm{CCD}$ image, but now stretched to show the faintest emission in the outer parts of the nebulosity. There is a faint, extended diffuse envelope almost $20^{\prime \prime}$ in diameter surrounding the already known, $10^{\prime \prime}$ diameter shell. It is tempting to suggest that this is outflowing gas from previous eruptions.
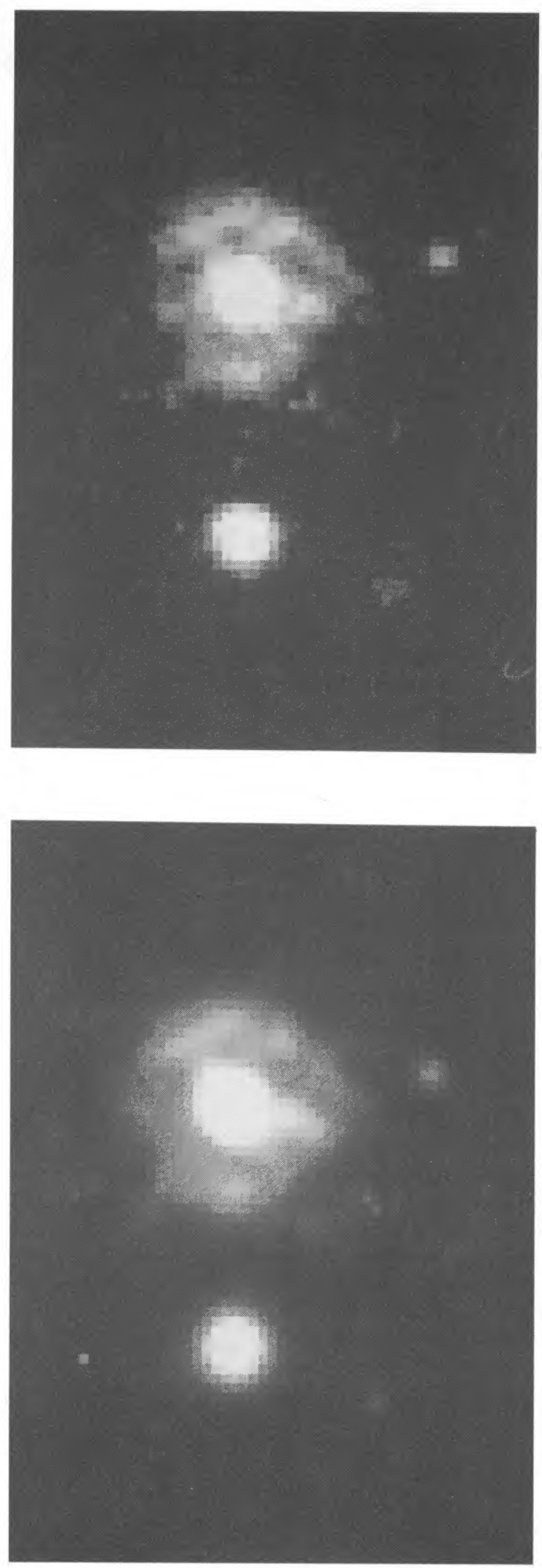

FIG. 1.- (a) Digitized image of T Pyx, taken from a 1980 March $\mathrm{H} \alpha+$ [N II] CTIO $4 \mathrm{~m}$ prime focus plate. (b) CCD image of T Pyx, in 1985, taken with an $\mathrm{H} \alpha+[\mathrm{N}$ II $]$ filter and the $3.9 \mathrm{~m} \mathrm{AAT}$. 
We also display, in Figure $2 b$, a broad-band $\mathrm{R}$ image of $\mathrm{T}$ Pyx. The regions of brightest $\mathrm{H} \alpha$ emission north and south of the central star are barely discernable.

The $\mathrm{H} \alpha+[\mathrm{N}$ II $]$ image of the ejecta has been constructed by subtracting a (suitably scaled) point spread function of a nearby field star from T Pyx in Figure $2 a$. The resultant net $\mathrm{H} \alpha+[\mathrm{N}$ II $]$ image is displayed in Fig. $2 c$. The bright star 13" south of T Pyx has also had a scaled PSF subtracted. Cuts through $\mathrm{T}$ Pyx and the bright star in Figures $2 a$ and $2 c$ are shown in Figure $2 d$ to demonstrate that almost all the continuum light has been subtracted form the T Pyx image in Figure $2 c$ (see the quantitative discussion below). The lumpy nature of the previously known shell is particularly apparent (as it is in Fig. $1 a$ and $1 b$ ). Isointensity maps of the R image (Fig. $2 b$ ) and the $\mathrm{H} \alpha+[\mathrm{N} \mathrm{II}]$ image (Fig. $2 a$ ) are shown in Figures $3 b$ and $3 a$, respectively, to again emphasize the reality and extent of the outer envelope.

The strong [O III] $\lambda 5007$ emission seen in Williams' (1982) spectra of the northern portion of the T Pyx shell prompted us to image T Pyx in the light of [O III]. Figure $4 a$ shows the [O III $\lambda 25007 \AA$ halo which surrounds T Pyx (see also Duerbeck 1987). A continuum B image is shown in Figure $4 b$, the net [O III] image ([O III]-B) is shown in Figure $4 c$, and an isointensity map of Figure $4 c$ is given in Figure 5. The region of [O III] emission is clearly smaller in extent than that of $\mathrm{H} \alpha+[\mathrm{N} \mathrm{II}]$, and considerably smoother.

Duerbeck and Seitter (1979) and Williams (1982) suggested that the bright T Pyx shell was ejected during the 1966 eruption, though they could not rule out the 1944 outburst. Seitter (1986) and Duerbeck (1987) now suggest that the 1966 shell is still unresolved, and that the main, bright shell is due to the 1944 eruption. With our new data in hand it was expected to be straightforward to measure the bright shell expansion and deduce the year of the shell's origin. In the event of a 1966 shell origin, the uniformly expanding shell should appear about $(1985-1980) /(1980-1966) \sim 36 \%$ larger in 1985 than in 1980. For a 1944 origin, the expected relative size increase is (19851980)/(1980-1944) $\sim 14 \%$. When we blinked the scaled and aligned images of Figures $1 a$ and $1 b$ (1980 and 1985, respectively) we could detect no expansion of the shell whatsoever. Seitter (1986) has also failed to detect expansion of T Pyx's shell.

In an attempt to quantify this unexpected result we have measured the angular distance of maximum $\mathrm{H} \alpha+[\mathrm{N}$ II $]$ emission from the central star of $\mathrm{T}$ Pyx in $5^{\circ}$ sectorial bins around the central star. (The emission maximum was chosen to avoid uncertainties in transforming photographic densities to intensities for comparison with the CCD data). This was done for both the 1980 and 1985 images, and the result is shown in Figure 6. Figure 6 confirms the apparent lack of shell expansion, and quantifies any expansion to be less than $\sim 10 \%$. Thus a 1966 origin of a (uniformly expanding) shell is clearly not consistent with our data while a 1944 origin seems just possible.

Inspection of Figure $2 d$ lends support to the suggestions of Seitter (1986) and Duerbeck (1987) that the bright shell $\left(\sim 10^{\prime \prime}\right.$ in diameter) is from the 1944 eruption. This is because of the brightness peaks $\sim 2^{\prime \prime}$ from the central star seen in Figure $2 d$, which may represent the (barely resolved) 1966 shell.

The reality of the brightness peaks is supported by comparison of the "before PSF subtraction" and "after PSF subtraction" traces through T Pyx and through the comparison star. The comparison star has practically disappeared in
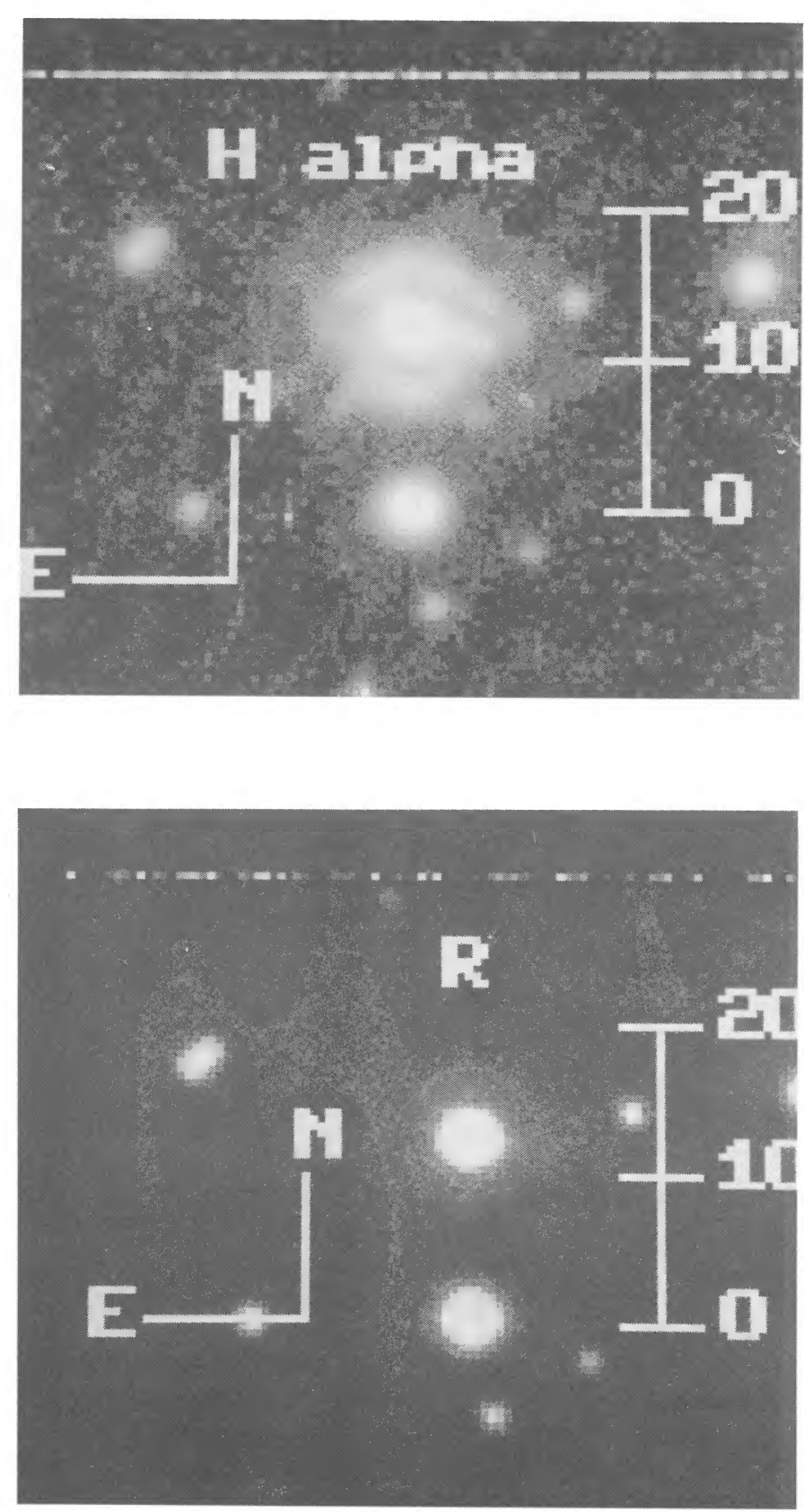

FIG. 2.-(a) Same as Fig. 1b, but stretched in intensity to show the faintest emission in the outer parts of the nebulosity. (b) Same as Fig. $1 b$, but through a broad-band $\mathrm{R}$ filter. (c) The net $\mathrm{H} \alpha+[\mathrm{N}$ II] image produced by subtracting a suitably scaled point spread function (taken from a star in the same image) from T Pyx and from the bright star south of T Pyx. (d) Intensity cuts through $\mathrm{T}$ Pyx and a bright star 13" south before (solid line) and after (dashed line) subtraction of a scaled point spread function from both. The peak (offscale) intensities of T Pyx and the comparison star are, respectively, 15,000 and 10,000 intensity units.

Figures $2 c$ and $2 d$. From a peak intensity of 10,000 units before PSF subtraction, only 50 units above sky remain after PSF subtraction. This is very different for $\mathrm{T}$ Pyx, dropping from 15,000 units to 500 intensity units (for the 2 " peaks).

If the $2^{\prime \prime}$ radius ring around T Pyx corresponds to the 1966 ejecta, then the $5^{\prime \prime}$ radius ring probably arose in the 1944 eruption, and the other faint shell is due to the 1920 (and possibly earlier) outbursts. 


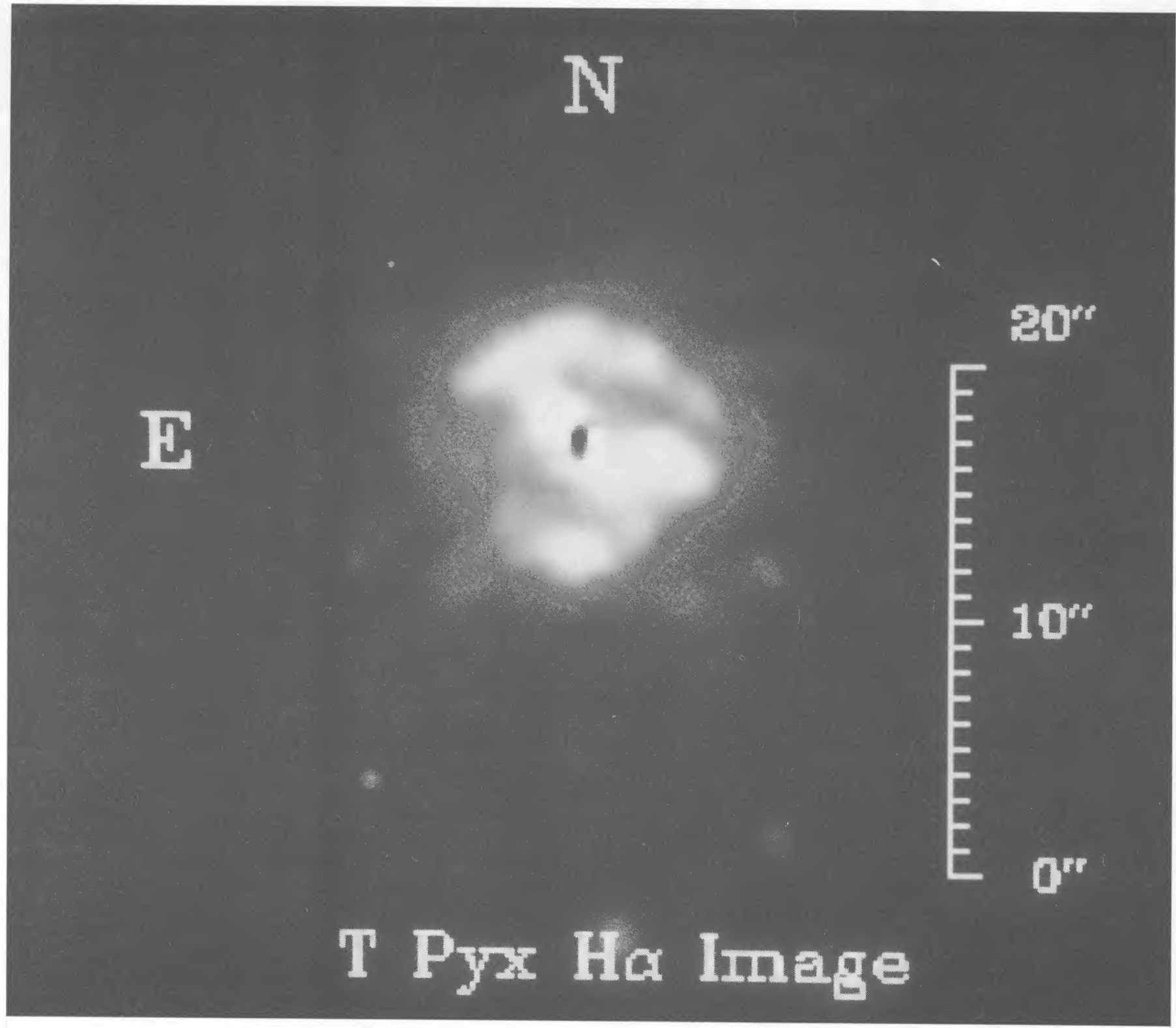

Fig. 2-Continued

\section{b) Spectra}

In Figure $7 a$ we display a spectrum of the shell of T Pyx. The spectral region covered is 6317 to $6972 \AA$ at $0.811 \AA$ pixel $^{-1}$ with a two-pixel FWHM (corresponding to a velocity resolution of $75 \mathrm{~km} \mathrm{~s}^{-1}$ ). The $\mathrm{H} \alpha$ and especially the [N II] lines dominate the shell spectrum. Figure $7 a$ shows all 800 pixels of the CCD (covering a $655 \AA$ spectral range), but stretched by a factor of 3 along the slit to emphasize the shell like appearance of the emission lines. Several cosmic-ray hits are visible as "hot" pixels. Figure $7 b$ is magnified view of 250 pixels centered at $6580 \AA$. The scale along and perpendicular to the slit is the same.

The [N II] 6584 line shows a shell-like expansion profile, with an expansion velocity of $350 \mathrm{~km} \mathrm{~s}^{-1}$. This $350 \mathrm{~km} \mathrm{~s}^{-1}$ expansion velocity is much less than the $850 \mathrm{~km} \mathrm{~s}^{-1}$ and 2000 $\mathrm{km} \mathrm{s}^{-1}$ velocities reported by Catchpole (1969) for the 1966 eruption. No spectroscopic velocities have been reported for the 1944 outburst. If the present, low expansion velocity is for 1966 ejecta then (1) most of its bulk kinetic energy must have been lost by interaction with circumstellar material, or (2) significant amounts of (now visible) low velocity material were ejected during the last outburst, or both of the above. Simulations of thermonuclear runaways on white dwarfs imply a wide range of ejection velocities, usually decreasing as the nova ejection process nears exhaustion. This agrees with the observed decrease with time of the observed ejection velocities of erupting novae.

If the present, low expansion rate refers to 1944 ejecta then nothing definitive can be said about deceleration of the ejecta. 


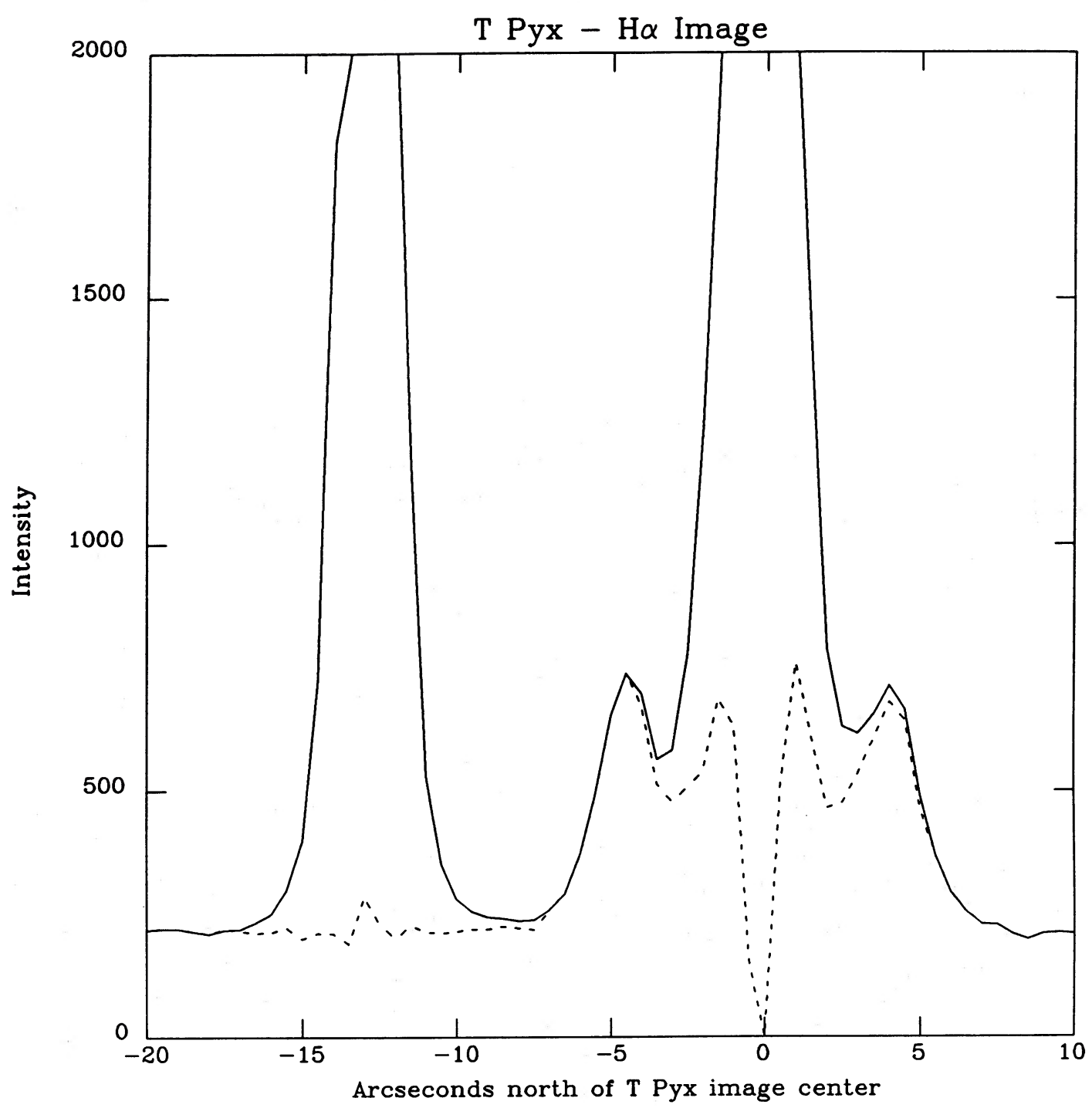

FIG. 2-Continued

\section{INTERPRETATION}

\section{a) Shell Expansion}

Webbink et al. (1987) reviewed the available evidence and concluded that T Pyx could lie anywhere from 1050 to 4500 pc from the Sun. Material ejected at $850 \mathrm{~km} \mathrm{~s}^{-1}$ from an object at $1 \mathrm{kpc}$ traverses 0.85 in 5 yr. Figure 6 implies that either T Pyx is significantly farther away than $1 \mathrm{kpc}$, or the ejection velocity is significantly less than $850 \mathrm{~km} \mathrm{~s}^{-1}$. The $350 \mathrm{~km} \mathrm{~s}^{-1}$ shell velocity reported here is consistent with a $1 \mathrm{kpc}$ distance and a 1944 generation of the bright shell, but not with a 1966 origin. Larger distances imply even older origin dates of the bright shell.

\section{b) A Planetary Nebula?}

The shell around T Pyx has several unique features that distinguish it from other nova shells: its image shows no obvious sign of expansion, and the spectrum is very similar to that of a planetary nebula.
In addition, there is a very low surface brightness outer envelope that surrounds the main shell. All of these characteristics are common to planetary nebulae, in addition to the fact that the shell emission appears both larger and less homogeneous in the radiation of lines of lower ionization species, such as $[\mathrm{N}$ II], than in the light of [O III]. Given the fact that some planetary nebulae, e.g., UU Sge + Abell 63 and Abell 41, do have central stars which are known to be binaries with periods which are comparable to those of cataclysmic variables (Bond, Liller, and Mannery 1987; Grauer and Bond 1983), the possibility that the T Pyx shell may be a planetary nebula should be considered. In fact, it has been suggested that mass-transfer binaries evolve from wider, longer period systems, and the ejection of a planetary nebula may sometimes be part of the evolutionary process that results in a CV (Ritter 1986).

A limit to the mass of the emitting shell can be set from the observed $\mathrm{H} \beta$ flux and the distance to the system. Suppose that the shell consists of gas with electron density $n_{e}$ filling a fraction $\epsilon$ of the emitting volume. The observed $H \beta$ flux from the 


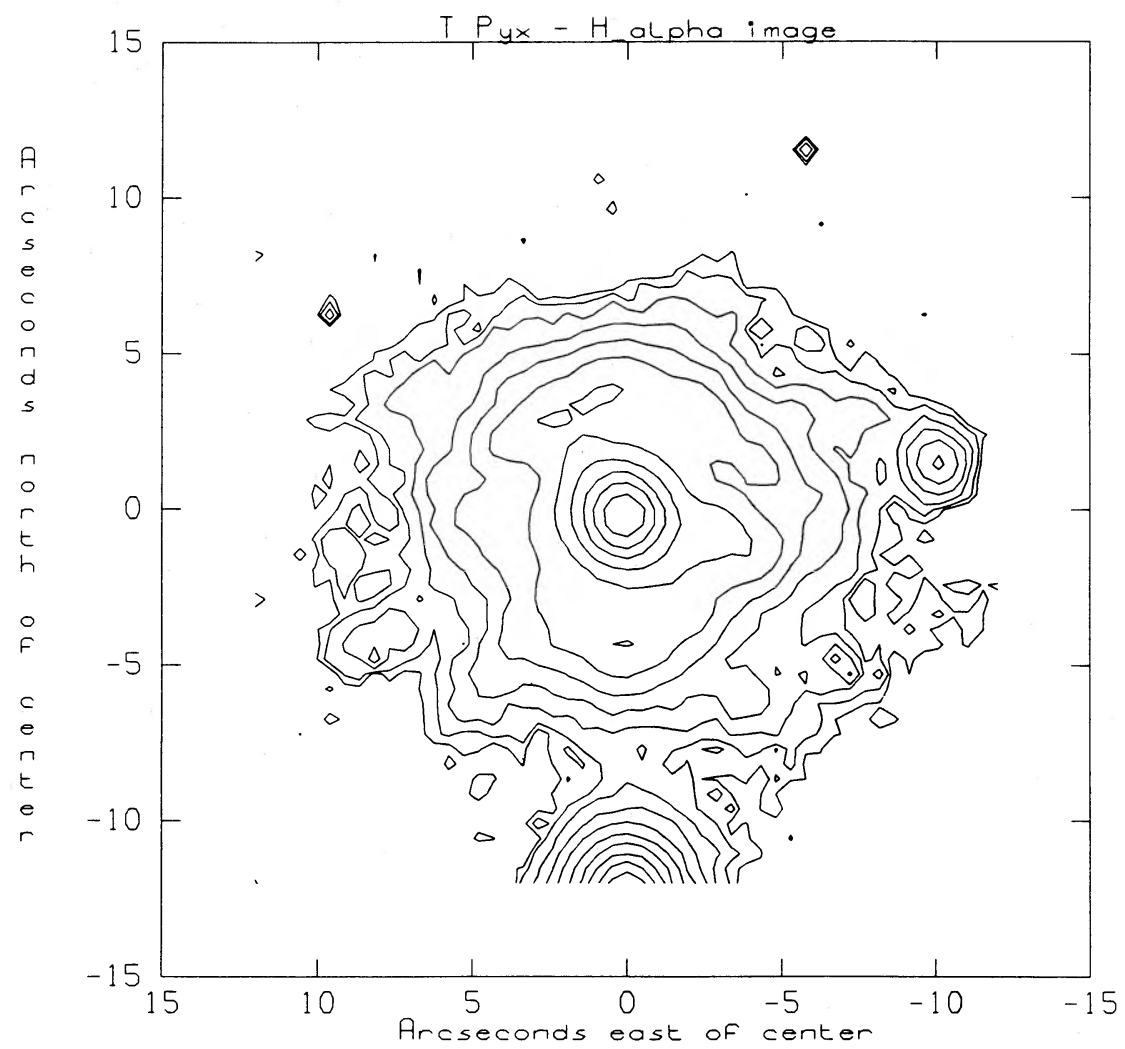

Fig. $3 a$

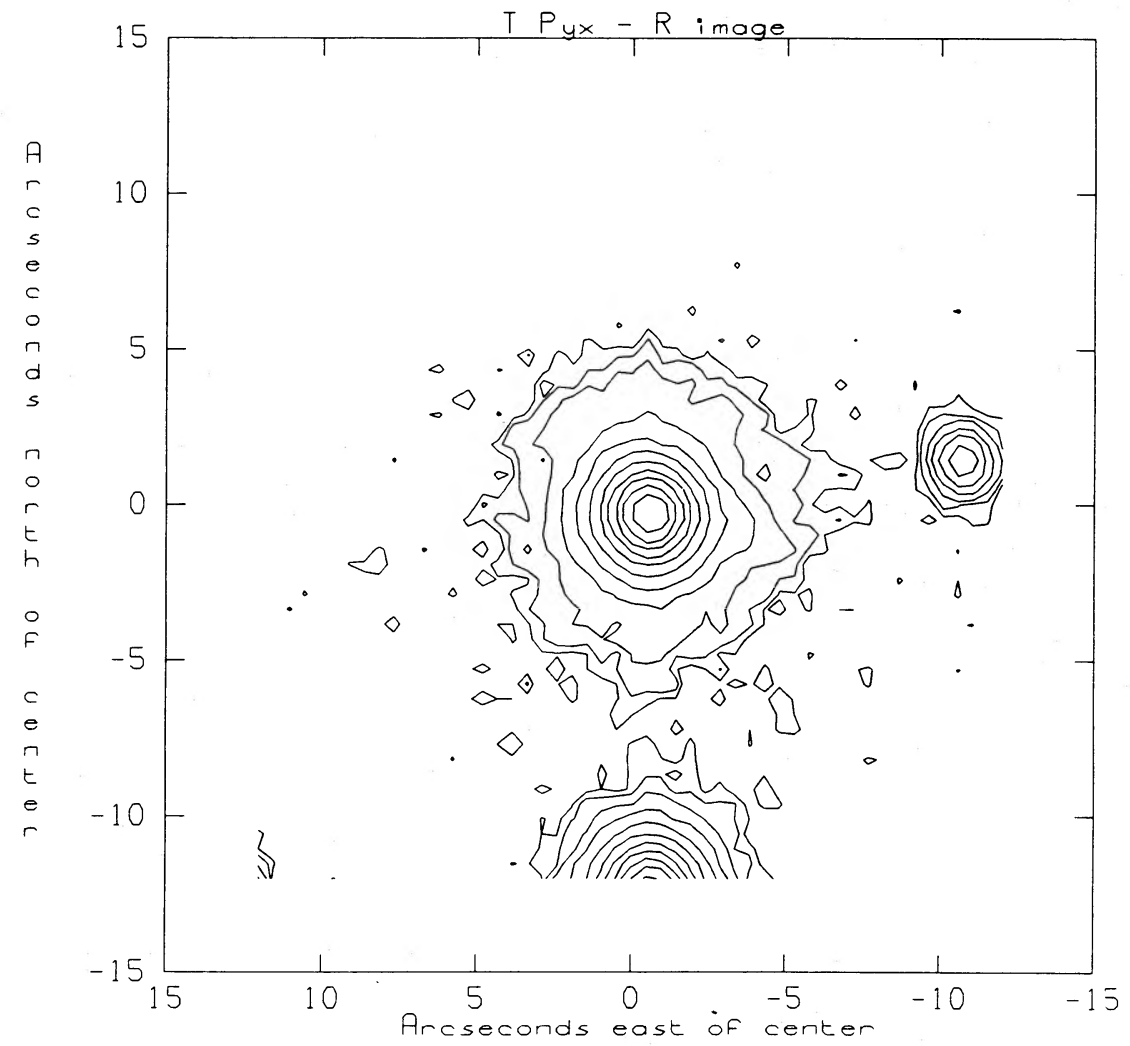

Fig. $3 b$

Fig. 3.- (a) An $\mathrm{H} \alpha+[\mathrm{N}$ II] isointensity map produced from Fig. 2a. Each contour is half the intensity of the next innermost contour. (b) An $R$ isointensity map produced from Fig. $2 b$. Contour intervals as in Fig. $3 a$. 

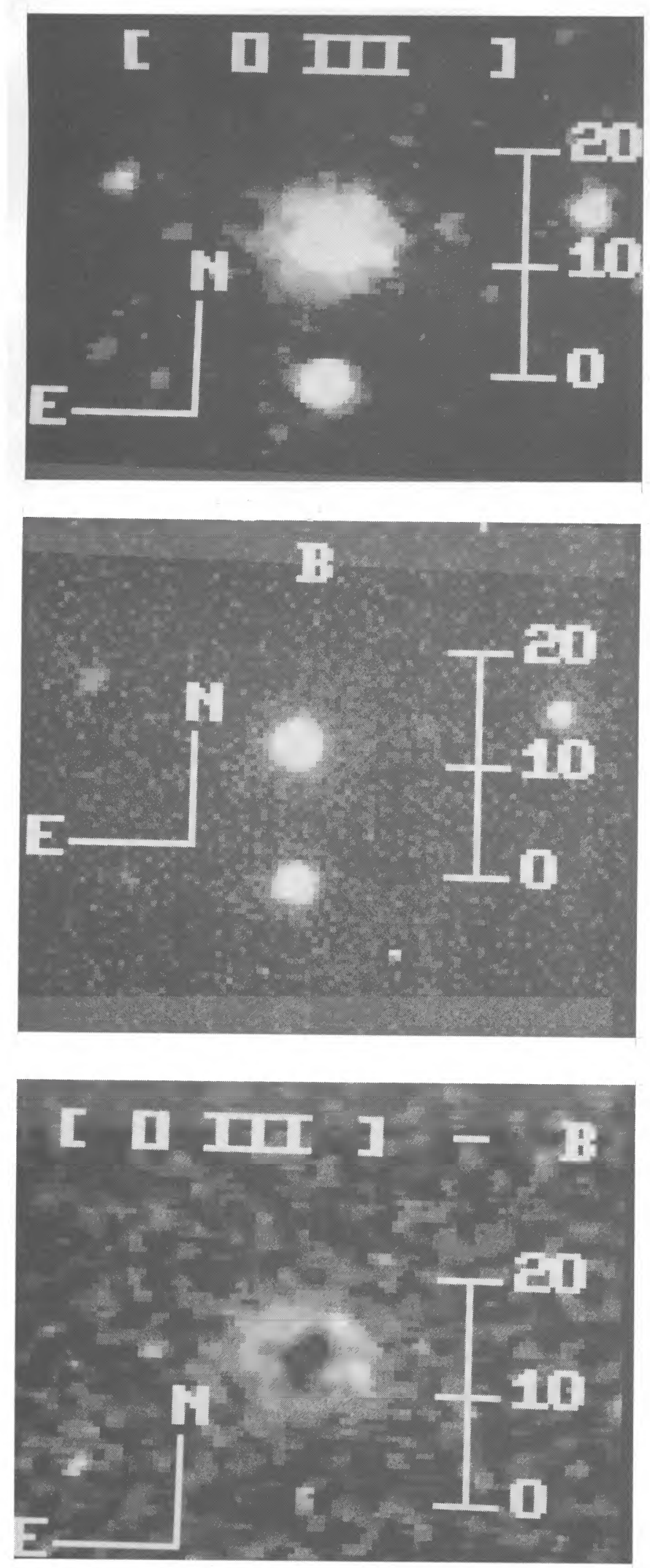

Fig. 4. - (a) An [O III] $\lambda 5007$ image of T Pyx, taken through a $20 \AA$ FWHM filter, with the $0.9 \mathrm{~m}$ telescope + RCA CCD at CTIO. (b) Same as (a) but with a broad-band $\mathrm{B}$ filter. $(c)$ The net [O III] difference image produced by aligning $(a)$ and $(b)$ and scaling in intensity to subtract out the stars outside the nebula. shell is then (Osterbrock 1974)

$$
F_{\mathrm{H} \beta}=\frac{4 \pi}{3 d^{2}} n_{e}^{2} \alpha_{\mathrm{H} \beta}^{\mathrm{eff}}\left(T_{e}\right) h v_{\beta} \in R_{n}^{3},
$$

where $d$ and $R_{n}$ are the distance and radius of the shell, and $\alpha_{\mathrm{H} \beta}^{\mathrm{eff}}$ is the effective recombination coefficient of $\mathrm{H} \beta$. The mass of the shell $M_{n}=(4 \pi / 3) m_{\mathrm{H}} n_{e} \in R_{n}^{3}$, therefore

$$
M_{n}=\left(\frac{4 \pi m_{\mathrm{H}}^{2} \epsilon}{3 h v_{\beta} \alpha_{\mathrm{H} \beta}} \theta_{0}^{3} d^{5} F_{\mathrm{H} \beta}\right)^{1 / 2},
$$

where $\theta_{0}=R_{n} / d=5^{\prime \prime}$ is the angular radius of the shell. Using a distance of $1.5 \mathrm{kpc}$ (based on $\mathrm{Ca}$ II and absolute magnitudedecline times, i.e., independent of shell expansion assumptions; Payne-Gaposchkin 1957; Catchpole 1969) and the measured flux for the shell of $F_{\mathrm{H} \beta}=1.2 \times 10^{-14} \mathrm{ergs} \mathrm{cm}^{-2} \mathrm{~s}^{-1}$ (Williams 1982), the requirement that $\epsilon<1$ leads to the limit that the emitting mass $M_{n} \lesssim 1 \times 10^{-4} M_{\odot}$. Since the emitted $\mathrm{H} \beta$ flux is more sensitive to density than the shell mass, any inhomogeneities drive this mass limit downward. The limit to the shell mass is therefore similar to the masses of nova shells, and much smaller than the typical mass of a planetary nebula, $M_{\mathrm{PN}} \sim 0.3$ $M_{\odot}$ (although masses smaller than $0.01 M_{\odot}$ in ionized gas are known). Therefore, on the basis of the mass of the envelope around T Pyx we can conclude that this is probably not a form of planetary nebula. The conclusion is made firm by $T$ Pyx's shell expansion velocity; $350 \mathrm{~km} \mathrm{~s}^{-1}$ is one order of magnitude too fast for a planetary nebula shell.

The emitting gas of the $10^{\prime \prime}$ diameter shell is expanding with a speed commensurate only with the 1944 or earlier eruptions. If the $10^{\prime \prime}$ shell is due to the 1966 outburst, then either the shell expansion has been retarded, or the emitting gas represents a standing shock through which the outflowing gas passes. In either case, there must be a surrounding medium to cause the phenomenon. Since the distance and galactic coordinates of $\mathrm{T}$ Pyx $\left(l=256^{\circ}, b=+9^{\circ}\right)$ place it far above the plane, the presence of sufficient ambient material to stop the expansion or produce a stationary shock is unexpected. Circumstellar gas (from an earlier eruption) could, however, produce deceleration. Sufficient kinematic energy must be converted to thermal energy in both cases that the line spectrum of collisionally shock-excited gas should be observed, i.e., emission lines of $\left[\mathrm{O}_{\mathrm{I}}\right] \lambda 6300$ and $\left[\mathrm{S}_{\mathrm{II}}\right] \lambda \lambda 6717,34$ should be produced at strengths comparable to the $[\mathrm{O} \mathrm{II}]$ and $[\mathrm{O} \mathrm{III}]$ lines. These $[\mathrm{O}$ I] and [S II] lines are not present in Williams' (1982) spectra. Again, this supports the 1944 identification of the $10^{\prime \prime}$ diameter shell. We don't know why no trace of the $850 \mathrm{~km} \mathrm{~s}^{-1}$ and 2000 $\mathrm{km} \mathrm{s}^{-1}$ velocities reported by Catchpole (1969) is seen.

Phenomenologically, the shell appears to be slowly expanding, photoionized gas having roughly solar abundances, from the similarity of its spectrum to that of planetary nebulae. That the observed abundances are roughly solar is in accord with theoretical work that suggests that (1) the white dwarfs in thermonuclear-powered RN must be massive, and the accreted shells must be of low mass (Nariai and Nomoto 1979); and (2) CNO enrichment of the accreted envelope by diffusion cannot occur in only a few decades (Kovetz and Prialnik 1985), (although hydrodynamic shear mixing processes might act this quickly-Livio and Truran 1987).

\section{c) How to Proceed}

Higher spatial resolution and longer temporal baseline observations are essential if we are to unambiguously identify 

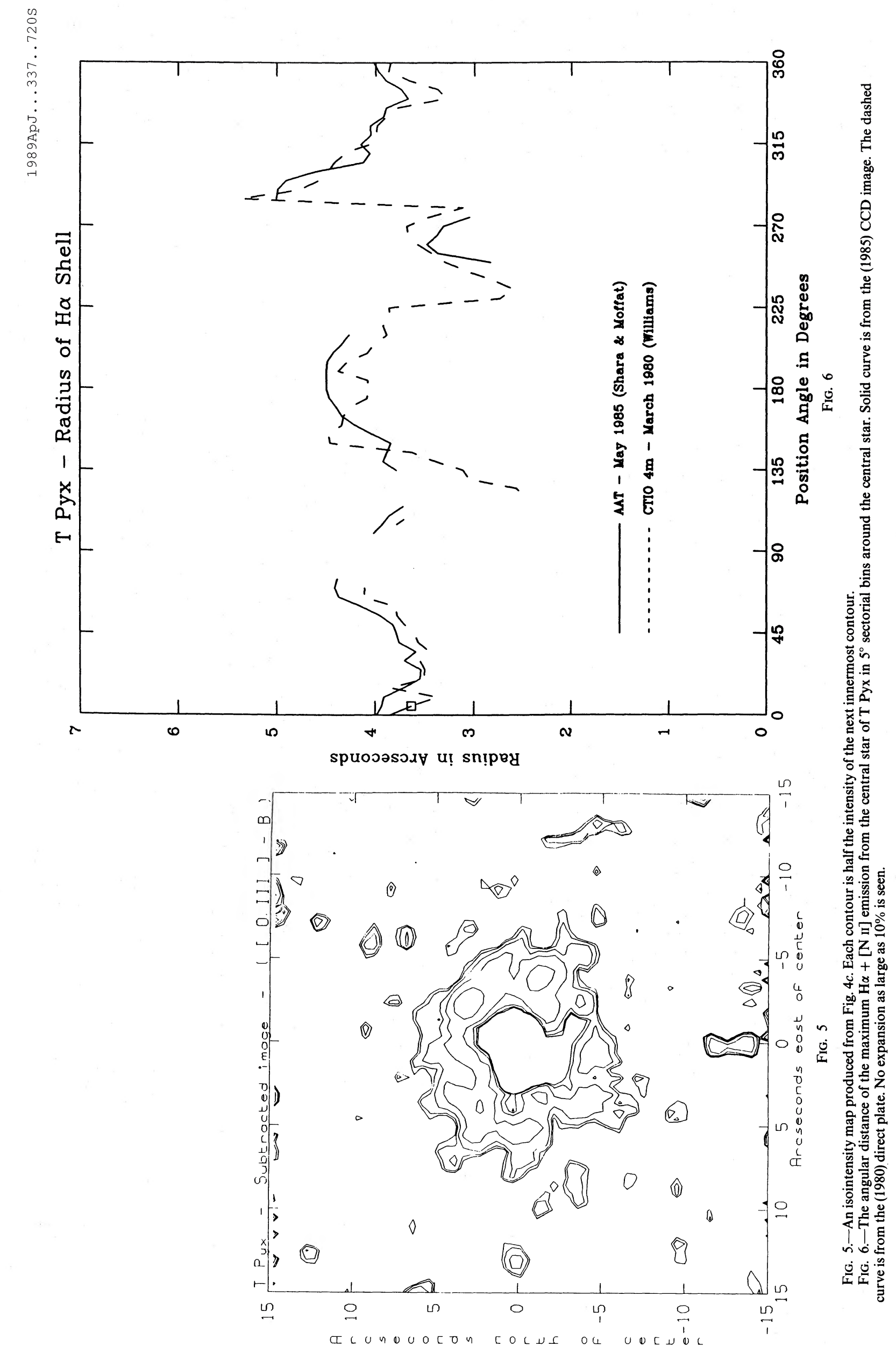

727

(C) American Astronomical Society - Provided by the NASA Astrophysics Data System 


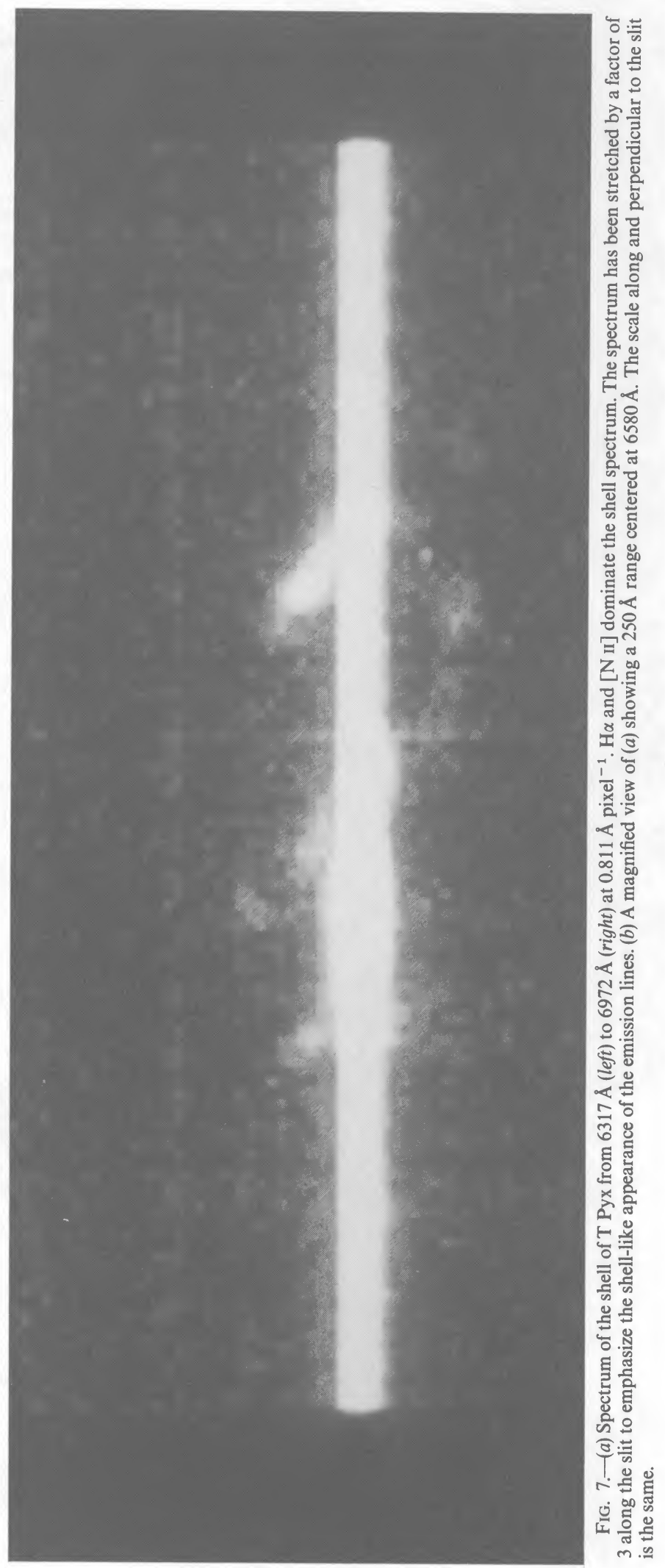


T Pyx' shells with particular outbursts. Over the next decade the $10^{\prime \prime}$ shell should show expansion if it arose in 1944, as should the 2 " radius ring. The next T Pyx outburst (due soon) should be observed every few days with the Hubble Space Telescope to watch for a light echo, which could yield more information about the distribution of matter about this object.

\section{SUMMARY}

We briefly summarize our results as follows:

1). A deep $\mathrm{H} \alpha+[\mathrm{N}$ II $]$ CCD image of the shell of the recurrent nova $\mathrm{T}$ Pyx reveals a faint, extended halo twice as large as the previously known shell.

2). An [O III $]$ image of $\mathrm{T}$ Pyx reveals a smoother, smaller morphology than that of the $\mathrm{H} \alpha+[\mathrm{N}$ II $]$ shell.

3). The bright $\mathrm{H} \alpha+[\mathrm{N}$ II] shell has expanded by less than $\sim 10 \%$ between 1980 and 1985, ruling out a 1966 formation date for a uniformly expanding shell. Spectra of the shell show an expansion velocity of $350 \mathrm{~km} \mathrm{~s}^{-1}$, much slower than the velocities observed by Catchpole (1969) shortly after the 1966 eruption. A PSF subtracted from an $\mathrm{H} \alpha+[\mathrm{N}$ II $]$ image of $\mathrm{T}$
Pyx reveals a 2 " radius ring around the central star, which may be the 1966 ejecta. If this suggestion (of Seitter 1986 and Duerbeck 1987) is correct, then the bright 5 " radius ring was ejected in 1944, and the faint outer shell is the remnant of the 1920 (and perhaps earlier) eruption.

4. The shell mass is less than $10^{-4} M_{\odot}$, ruling out a planetary nebula-like origin. The observed shell is slowly expanding photoionized gas of roughly solar abudance.

5. T Pyx's distance is still very uncertain. High temporal and spatial resolution observations of the next eruption are crucial to understanding T Pyx and its shells.

We are pleased to thank D. Lee and D. Allen for support at the AAT, and M. Navarrete for support at CTIO. Mike Potter produced the PDS scan of the CTIO plate, as well as the isointensity maps and T Pyx pictures. We also thank D. Klinglesmith for support in use of the PDS microdensitometer of the Goddard Space Flight Center, and V. Trimble for useful suggestions. J. G. C. is grateful for support from the Caltech Recycling Center.
Bond, H. E., Liller, W., and Mannery, E. J. 1978, Ap. J., 223, 252.

Catchpole, R. M. 1969, M.N.R.A.S., 142, 119.

Duerbeck, H. W. 1987, ESO Messenger, No. 50, 8.

Duerbeck, H. W., and Seitter, W. C. 1979, ESO Messenger, No. 17, 3.

Grauer, A., and Bond, H. E. 1983, Ap. J., 271, 259.

Kovetz, D., and Prialnik, D, 1985, Ap. J., 291, 812

Livio, M., and Truran, J. 1987, preprint.

Mayall, M. W. 1967, J.R.A.S. Canada, 61, 349.

Nariai, K., and Nomoto, K. 1979, in IAU Colloquium 53, White Dwarfs and

Variable Degenerate Stars, ed. H. M. Van Horn and V., Weidemann

(Rochester: University of Rochester), p. 525.

Osterbrock, D. 1974, Astrophysics of Gaseous Nebulae (San Francisco:

Freeman).

\section{REFERENCES}

Patterson, J. 1984, Ap. J. Suppl., 54, 443.

Payne-Gaposchkin, C. 1975, The Galactic Novae (Amsterdam: North Holland).

Ritter, H. 1986, Astr. Ap., 169, 139.

Seitter, W. C. 1986, in RS Ophiuchi (1985) and the Recurrent Nova Phenomenon, ed. M. F. Bode (Amsterdam: VNU), p. 63.

Shara, M. M., Livio, M., Moffat, A. F. J., and Orio, M. 1986, Ap. J., 311, 163.

Webbink, R. F., Livio, M., Truran, J. W., and Orio, M. 1986, Ap. J., 314, 653. Williams, R. E. 1981, Sci. Am., 244, 120.

- 1982, Ap. J., 261, 170 .

Judith G. CoHEN : California Institute of Technology, 105-24, Pasadena, CA 91125

Anthony F. J. Moffat: Département de Physique, Université de Montréal, C.P. 6128 Succ “A,” Montréal, Québec H3C 3J7, Canada

Michael M. Shara: Space Telescope Science Institute, 3700 San Martin Drive, Baltimore, MD 21218

RoBert E. WiLliams Cerro Tololo Interamerican Observatory, Casilla 603, La Serena, Chile 